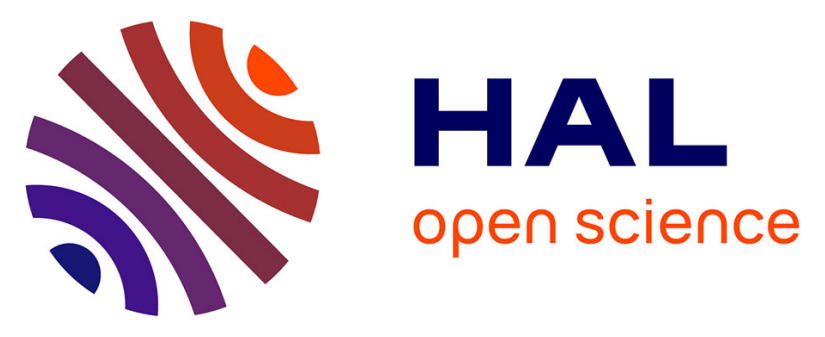

\title{
Suivi d'endommagement par EA dans un composite obtenu par pultrusion et soumis à un chargement en flexion 3 points
}

Jamal Fajoui, Nicolas Terrien, Fethi Dahmene, Aurélie Cordelle, Xavier Chapeleau, Monssef Drissi-Habti

\section{To cite this version:}

Jamal Fajoui, Nicolas Terrien, Fethi Dahmene, Aurélie Cordelle, Xavier Chapeleau, et al.. Suivi d'endommagement par EA dans un composite obtenu par pultrusion et soumis à un chargement en flexion 3 points. Revue des composites et des matériaux avancés $=$ Journal of Composite and Advanced Materials, 2013, 2 (23), pp. 331-334. 10.3166/rcma.23.331-344 . hal-00867223

\section{HAL Id: hal-00867223 https://hal.science/hal-00867223}

Submitted on 27 Sep 2013

HAL is a multi-disciplinary open access archive for the deposit and dissemination of scientific research documents, whether they are published or not. The documents may come from teaching and research institutions in France or abroad, or from public or private research centers.
L'archive ouverte pluridisciplinaire HAL, est destinée au dépôt et à la diffusion de documents scientifiques de niveau recherche, publiés ou non, émanant des établissements d'enseignement et de recherche français ou étrangers, des laboratoires publics ou privés. 


\title{
Suivi d'endommagement par EA dans un composite obtenu par pultrusion et soumis à un chargement en flexion 3 points
}

\author{
$* * * * * * * * * * * * * * *$ \\ Jamal Fajoui, ${ }^{1}$, Nicolas Terrien ${ }^{2}$, Fethi Dahmène ${ }^{2}$, Aurélie Cordelle ${ }^{1}$, Xavier \\ Chapeleau ${ }^{1}$, Monssef Drissi-Habti ${ }^{1}$ * \\ PRES LUNAM, IFSTTAR, Département Mesure, Auscultation et Calcul Scientifique \\ (MACS), Route de Bouaye, CS 0544 344, Bouguenais cedex \\ * Pour toute correspondance : monssef.drissi-habti@ifsttar.fr \\ RÉSUMÉ : \\ Dans ce travail, un matériau composite à fibres de verre et à matrice vinylester, obtenu par \\ pultrusion, est soumis à un chargement en flexion 3 points, d'abord sous chargement quasi- \\ statique et ensuite, en fatigue à fréquence relativement faible. La technique d'émission \\ acoustique est utilisée lors des essais afin, de préciser le seuil d'endommagement et \\ également de suivre son développement, lors des essais. Les résultats ont montré que le seuil \\ d'endommagement survient bien avant la fin de proportionnalité. Ce résultat a permis de bien \\ préciser l'intervalle de contrainte le long duquel les essais de fatigue sur ce matériau seront \\ pratiqués; à savoir entre la contrainte seuil d'apparition d'endommagement et la contrainte \\ de fin de proportionnalité. Jusqu'à la fin de proportionnalité, le scénario d'endommagement \\ du matériau étudié, suggère un mode d'endommagement, gouverné principalement par les \\ divers micro-mécanismes d'endommagement de la matrice (microfissuration de la matrice \\ longitudinalement et transversalement aux fibres). A niveaux de contrainte plus élevés, les \\ fibres commencent à se rompre, en nombre significatif, accompagnées d'un large éventail de \\ mécanismes d'endommagement. Ceci est prouvé par une distribution très élargie des \\ amplitudes des événements acoustiques.
}

ABSTRACT:

In this work, a composite material with glass fibers and vinylester matrix, obtained by pultrusion, is subject to a 3-point bending test, first under quasi-static loading and then to fatigue, at relatively low frequency. The acoustic emission technique is used during the tests in order, to clarify the damage threshold and also to monitor its development, during testing. The results showed that the damage threshold occurs well before the end of proportionality. This result allowed us to clearly specify the interval along which the stress fatigue tests have to be performed on this material, namely between the stress threshold for damage and the end constraint of proportionality. The scenario of damage to the material studied suggests a damage mode at low stress (up to the end of proportionality), governed by various microdamage mechanisms of the matrix (matrix microcracking longitudinally and transversely to fibers). At higher stress, the fibers begin to break, in significant numbers, along with a large

Revue. Volume $\mathrm{X}-\mathrm{n}^{\circ} \mathrm{x} /$ année, pages 1 à $\mathrm{X}$ 
diversity of damage mechanisms. This is proven by a very broad distribution of amplitudes of acoustic events.

MOTS-CLÉS : Matériau composite, endommagement, émission acoustique, fatigue, KEYWORDS: Composite material, damage mechanism, acoustic emission, fatigue

\section{Extended abstract}

Glass-fiber reinforced composite materials are increasingly used in structural applications. Their mechanical properties (low density, high strength and high chemical corrosion-resistance ...) offer interesting alternative solutions to metallic or concrete material. The pultrusion is a composite material processing technique that is penetrating the civil engineering market since the beginning of the 90's. It is a continuous process well adapted to mass production of linearly shaped profiles. Moreover, many research on the use of pultruded structural shapes for civil engineering building and bridge applications have shown the undeniable potential for the use of these products.

Reliability studies of civil engineering composites structures depending on damage tolerance are very important. Most of these structures should withstand a high number of cycles at elevated stress values, under various environmental conditions. The mechanical behavior of composite materials subjected to cycling loading is complex. Static and fatigue failure in pultruded composite materials exhibit different damage mechanisms such like matrix cracking, fiber-matrix debonding and fiber fractures. Performing fatigue testing, metallographic observation, as well analytical modelling are therefore essential to detect the onset of damage and to predict its development as a function of the applied stress.

In this contribution, the mechanical behavior of smart composite specimens under quasi-static and dynamic fatigue in 3-points bending is investigated experimentally. Acoustic emission technique is used to detect damage development and its propagation as a function of applied stress. Embedded optical fiber-based sensors are used for strain monitoring. The following 4 proposed sequences of damage allow a clear view of the mechanical behavior of the material. Each is dominated by a given damage mechanism. The first phase starts at the beginning of the trial and ends at the registration constraint of the first acoustic emission events. This part is in principle free from damage. The 2 nd phase starts at $e=0.22 \%$ and stops at the proportional limit. This phase shows multiple matrix damage, along with the failure of weakest fibers. The third phase begins at the end of proportionality and ends at the maximum stress. It is characterized by progressive failure of fibers in large numbers. The purpose of this part is reached when a critical number of fibers failures at the peak load. The final phase consists of an increase in mode II cracking. The sequences depicted above are useful in the sense that they allow a direct relationship between the mechanical behavior and the evolution of the microstructure.

Quasi-static tests, conducted along with acoustic emission records enabled the 
identification of damage mechanisms acting with increasing stress values. The definition of the stress range over which fatigue tests will be conducted was also identified. Results of fatigue tests showed that up to 186MPa, there is fairly no stiffness reduction. Beyond this stress level, fibers failures take place and therefore significant decrease of mechanical properties was recorded.

\section{Introduction}

L'utilisation des matériaux composites à matrices thermodurcissables est en forte croissance dans de nombreuses applications industrielles. Durant leurs vies en service, ces matériaux sont souvent exposés pour de longues périodes à des chargements mécaniques, souvent sous atmosphères très agressives. Ceci génère des endommagements qui peuvent conduire à la ruine prématurée de la structure. Ainsi, il est important de déceler avec précision l'initiation de l'endommagement, ainsi que ses séquences de déclenchement en fonction des niveaux de contraintes appliquées.

Expérimentalement, de nombreuses études se sont intéressées à l'initiation et à la propagation de l'endommagement dans des matériaux composites. Plusieurs approches ont été proposées : C'est le cas de la tomographie par rayons X [Carmona 2009], par ultrasons [Ducret, 2002], par corrélations d'images [Crouzeix, 2008].

Dans ce travail, nous proposons un couplage entre les essais mécaniques en flexion 3 points et les enregistrements d'émission acoustique, afin de détecter l'initiation et la propagation d'endommagement dans un composite à fibres de verre et à matrice vinylester. A travers une étude fine des différents mécanismes d'endommagement développés par le matériau composite étudié, notre but est de pouvoir définir un intervalle qui débute à la contrainte d'initiation de l'endommagement détectée par émission acoustique et se termine par celle de fin de proportionnalité. Cet intervalle qui correspond à un comportement mécanique réversible nous permettra de procéder à des essais de fatigue en flexion 3 points avec pour objectif de quantifier l'écart au comportement élastique en fonction du niveau de contrainte appliquée et de la fréquence des essais. Parallèlement, les enregistrements d'émission acoustique nous ont permis de discuter qualitativement des intervalles d'amplitude correspondant aux divers mécanismes d'endommagement développés par le matériau composite étudié, que ce soit lors d'essais quasi-statiques ou d'essais de fatigue

\section{Présentation des matériaux étudiés}

\subsection{Matrices et renforts}

Durant la fabrication d'un composite, le choix de la résine est très important. Il dépend de plusieurs critères : les propriétés mécaniques, la résistance à la rupture, la résistance à la fatigue, etc.

Dans cette étude, nos échantillons sont fabriqués à partir de fibres de verre et d'une matrice du type vinylester. Cette dernière présente, en particulier, de 
meilleures performances de protection chimique des fibres. Le Tableau 1 résume quelques propriétés mécaniques de la matrice étudiée. Les fibres, objets de ce travail sont celles en verre, du type E et le Tableau 2 en regroupe quelques propriétés. Après un traitement de désensimage, les fibres de verres peuvent être imprégnées par une résine pour constituer un matériau composite, via le procédé de pultrusion.

\begin{tabular}{|c|c|c|c|c|}
\hline & $\begin{array}{c}\text { Module } \\
\text { d'Young }(\mathrm{GPa})\end{array}$ & $\begin{array}{c}\text { Coefficient } \\
\text { de Poisson }\end{array}$ & $\begin{array}{c}\text { Contrainte de } \\
\text { rupture }(\mathrm{MPa})\end{array}$ & $\begin{array}{c}\text { Allongement } \\
\text { à rupture }(\%)\end{array}$ \\
\hline Vinylester & $2,5-3$ & 0,35 & 60 & $3-4$ \\
\hline
\end{tabular}

Tableau 1 : Propriétés mécaniques de la matrice vinylester monolithique

\begin{tabular}{|c|c|c|c|}
\hline $\begin{array}{c}\text { Module d'Young } \\
(\mathrm{GPa})\end{array}$ & $\begin{array}{c}\text { Coefficient de } \\
\text { Poisson }\end{array}$ & $\begin{array}{c}\text { Contrainte de } \\
\text { rupture }(\mathrm{MPa})\end{array}$ & $\begin{array}{c}\text { Allongement à } \\
\text { rupture (\%) }\end{array}$ \\
\hline 73 & 0,17 & 2,5 & 3,5 \\
\hline
\end{tabular}

Tableau 2 : Propriétés physiques de la fibre de verre $\mathrm{E}$

\subsection{Procédé de fabrication par pultrusion}

La pultrusion est un procédé de moulage des composites qui permet de fabriquer des pièces, à section constante, renforcées dans le sens longitudinal (Figure 1). Pour ce faire, les fibres de renfort (jusqu'à $70 \%$ en volume) sont «tirées », à travers un bain d'imprégnation, puis dans une filière chauffée, de manière à initier la réaction de réticulation (ou polymérisation). Puis, le profilé solide est refroidi suffisamment, de façon à pouvoir être pincé dans la «tireuse », sans risque de déformation. La vitesse de tirage varie de façon importante en fonction de la matrice utilisée (réactivité), des taux de renfort et de charge, de la section et de la complexité du profilé.

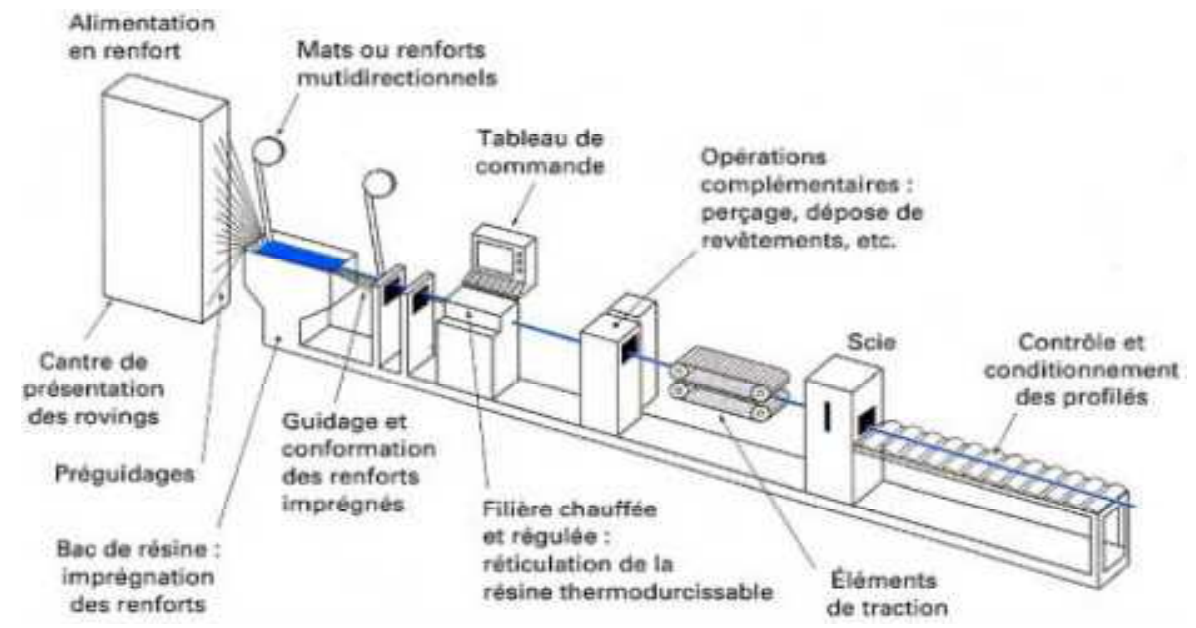

Figure 1 : Schéma de principe d'une ligne de pultrusion [Binse, Tech D’ing] 


\subsection{Composite à matrice vinylester et à fibres de verre}

Les composites étudiés présentent un taux de renforcement volumique de $66 \%$. Le Tableau 3 donne quelques propriétés mécaniques, calculées par la loi des mélanges, et par la méthode de transitions d'échelles, à l'aide du modèle autocohérent.

La Figure 2 illustre la microstructure du matériau composite à matrice vinylester et à fibres de verre, obtenu par microscopie électronique à balayage (MEB). La distribution de la section transversale (diamètre des fibres) et celle de la position des fibres de verres dans la matrice sont inhomogène (Figure 2.a). On note également que les fibres ne sont pas parfaitement rectilignes (Figure 2.b). Certaines fibres coupent le plan de l'image et d'autres font un angle plus au moins important avec la direction principale du composite (sens de pultrusion). Finalement, on observe la présence de porosité et des additifs dans le matériau.

\begin{tabular}{|l|c|c|c|}
\hline \multirow{2}{*}{} & \multicolumn{3}{|c|}{ Vinylester / fibres de verre } \\
\cline { 2 - 4 } & $\mathrm{E}_{11}(\mathrm{GPa})$ & $\mathrm{v}_{12}$ & $\mathrm{G}_{12}(\mathrm{GPa})$ \\
\hline $\begin{array}{l}\text { Loi des } \\
\text { mélanges }\end{array}$ & 50,44 & 0,26 & 3,18 \\
\hline $\begin{array}{l}\text { Auto- } \\
\text { cohérent }\end{array}$ & 50,47 & 0,23 & 2,71 \\
\hline
\end{tabular}

Tableau 3 : Propriétés mécaniques théoriques du matériau composite à matrice vinylester et fibres de verre

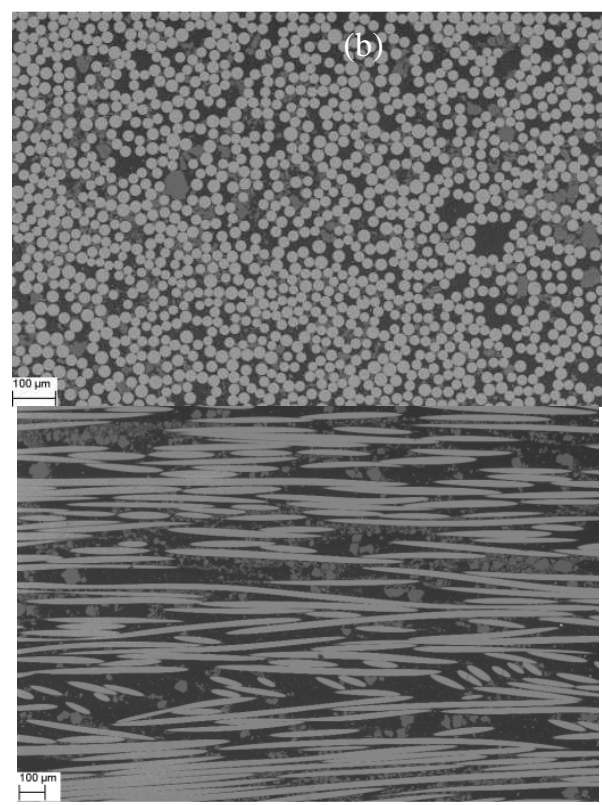


Figure 2 : Observations par le MEB de la microstructure du vinylester/Verre. On remarque le très fort taux volumique des fibres.

\section{Méthodes expérimentales}

Dans cette partie, nous présentons les conditions expérimentales des essais mécaniques et des enregistrements acoustiques.

Des éprouvettes tests, possèdant une section rectangulaire $\left(40 \times 16 \mathrm{~mm}^{2}\right)$, ont été fabriquées afin d'effectuer des essais de flexions 3 points, avec un écartement entre points d'appui de $260 \mathrm{~mm}$. Les essais ont été réalisés sur une machine MTS équipée d'une cellule de force de capacité $100 \mathrm{kN}$. Nous avons réalisé trois types d'essais de flexion 3 points :

- quasi-statiques jusqu'à rupture qui ont été effectués à une vitesse de déplacement imposée de $1 \mathrm{~mm} / \mathrm{min}$. Le couplage de ces essais avec les enregistrements des émissions acoustiques (EA) a permis de déterminer le début d'endommagement et le suivi de son évolution durant le chargement, ainsi que la prédiction de la zone de rupture.

- des essais de chargement - déchargement, à une vitesse de déplacement de 1 $\mathrm{mm} / \mathrm{min}$, avec des incréments de déplacement de $0,25 \mathrm{~mm}$, de la flèche. Les essais ont été arrêtés à un déplacement de $10 \mathrm{~mm}$ de la flèche. L'analyse des données d'émission acoustique a permis de déterminer avec plus de précision le début de l'endommagement dans les éprouvettes testées.

- des essais de fatigue pilotés en charge, à une fréquence de $3 \mathrm{~Hz}$ avec un signal du type rampe. Le choix de la valeur de fréquence permet d'éviter les émissions acoustiques dues aux nuisances sonores de la machine à une fréquence plus élevée. L'essai de fatigue s'est déroulé en 6 paliers de 36000 cycles. Le Tableau 4 donne les forces et les contraintes utilisées pour chaque palier. Tous les essais ont été effectués à température ambiante. Avec le suivi par émission acoustique, ces essais ont permis d'analyser l'endommagement des matériaux composites, en fatigue.

\begin{tabular}{|l|c|c|c|c|c|c|}
\hline & Palier 1 & Palier 2 & Palier 3 & Palier 4 & Palier 5 & Palier 6 \\
\hline Nombre de cycles & 36000 & 36000 & 36000 & 36000 & 36000 & 36000 \\
\hline Force maximale (KN) & 0,95 & 1,9 & 3,45 & 5,25 & 6,35 & 7,85 \\
\hline Déplacement maximale (mm) & 0,83 & 1,67 & 3,01 & 4,60 & 5,60 & 7,08 \\
\hline Contrainte maximale (MPa) & 36,1 & 73,3 & 131,4 & 200 & 241,8 & 300 \\
\hline Déformation maximale (\%) & 0,11 & 0,23 & 0,42 & 0,64 & 0,80 & 1,00 \\
\hline
\end{tabular}

Tableau 4 : Forces commandées et le déplacement/la contrainte/la déformation correspondant(e) durant l'essai de fatigue

Le suivi par émission acoustique, comme le montre la Figure 3, les enregistrements d'EA ont été effectués à l'aide de deux capteurs R15 positionnés à 
$80 \mathrm{~mm}$ du centre de l'éprouvette. Des préamplificateurs ont été branchés entre les capteurs et la carte d'acquisition, afin d'amplifier et adapter le signal en basse fréquence. Les capteurs ne mesurent que les EA créent entre eux (c.à.d. dans une zone de $160 \mathrm{~mm}$ ). Ceci a permis de minimiser les nuisances sonores de la machine. Pour détecter le tout début d'endommagement du matériau composite, nous avons choisi de travailler avec un seuil de $35 \mathrm{~dB}$. Ce seuil reste conforme aux résultats bibliographiques (Cf. par exemple, Drissi-Habti, 1995 ; Huguet, 2002).

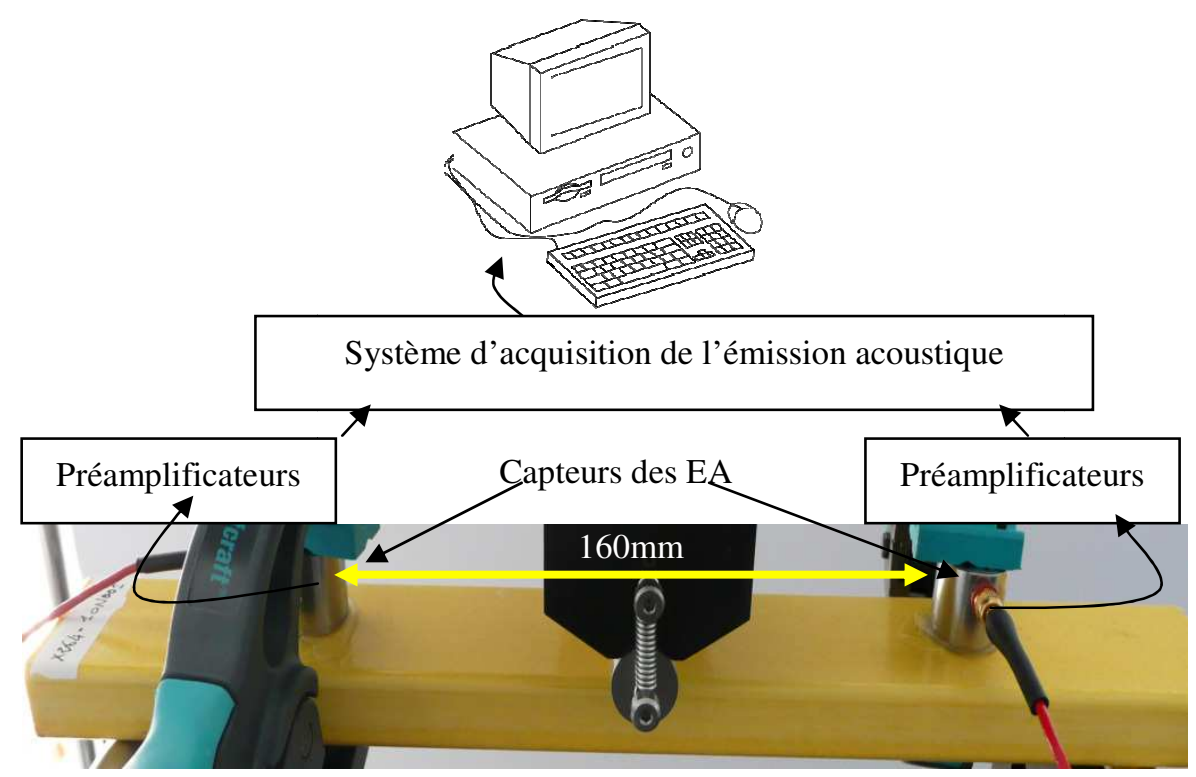

Figure 3 : Positionnement des capteurs sur les éprouvettes

\section{Résultats et discussions}

4.1. Modes d'endommagement et de ruptures des matériaux composites obtenus par pultrusion, soumis à une flexion 3 points - Relation avec l'activité acoustique

Les éprouvettes en matériaux composites à matrices polymères et à fibres continues, soumis à la flexion 3 points se comportent d'une façon très particulière.

La Figure 4 représente la courbe contrainte / déformation enregistrée lors de l'essai de flexion 3 points en quasi-statique, couplée à l'évolution du nombre de coups cumulés des émissions acoustiques (EA), en fonction de la déformation. L'analyse de cette courbe, couplée aux différentes observations microscopiques nous pousse à proposer le scénario suivant, qui semble le plus probable pour décrire l'endommagement et la ruine du matériau étudié. L'endommagement commence à faible niveau de contrainte $(75 \mathrm{MPa})$ et consiste en la mise en tension de ces fibres 
sur la face en traction (De façon générale, dans un matériau obtenu par pultrusion, les fibres ne sont pas alignées de façon très rectiligne), tout autant qu'un endommagement au niveau de la matrice. Cette valeur de début d'endommagement se retrouve également sur la courbe de flexion en mode chargement déchargement (Figure 5), où le premier endommagement est détecté à une déformation de $0,25 \%$. Au-delà de cette valeur seuil, les décollements fibre-fibre et les ruptures matricielles, parallèlement et transversalement aux fibres, sont responsables de l'accroissement du nombre de coups cumulés d'EA. Compte tenu d'une fraction volumique de fibres très élevée et d'une plus faible rigidité de la matrice, la fissuration matricielle dans ces matériaux n'engendre pas de comportement mécanique non linéaire prononcé. Dans ces conditions, pratiquement jusqu'aux environs de la limite de proportionnalité, la fissuration de la matrice: le long des fibres (suite aux décollements fibre-matrice) ainsi que la fissuration transverse sont a priori les mécanismes d'endommagement majeurs, responsables de l'activité acoustique. Bien évidemment, il doit y avoir des sources secondaires d'EA, en particulier quelques ruptures des fibres les plus faibles.

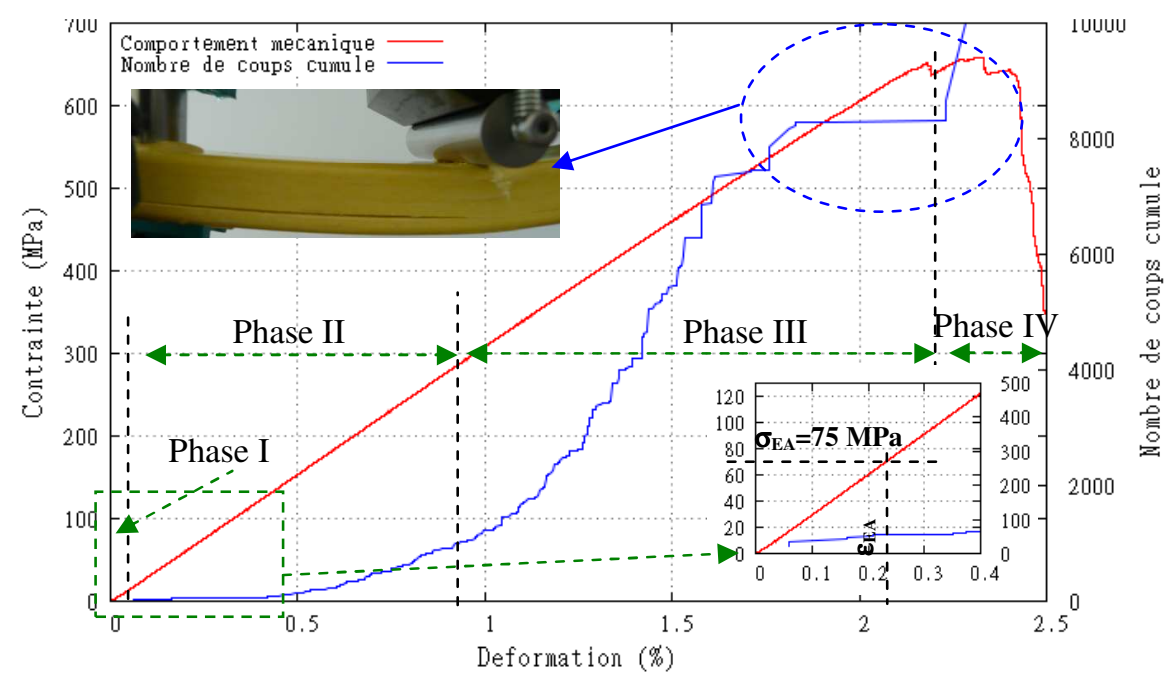

Figure 4 : Courbe de chargement en flexion 3 points, couplée au nombre d'alternances cumulées en cours d'essai 


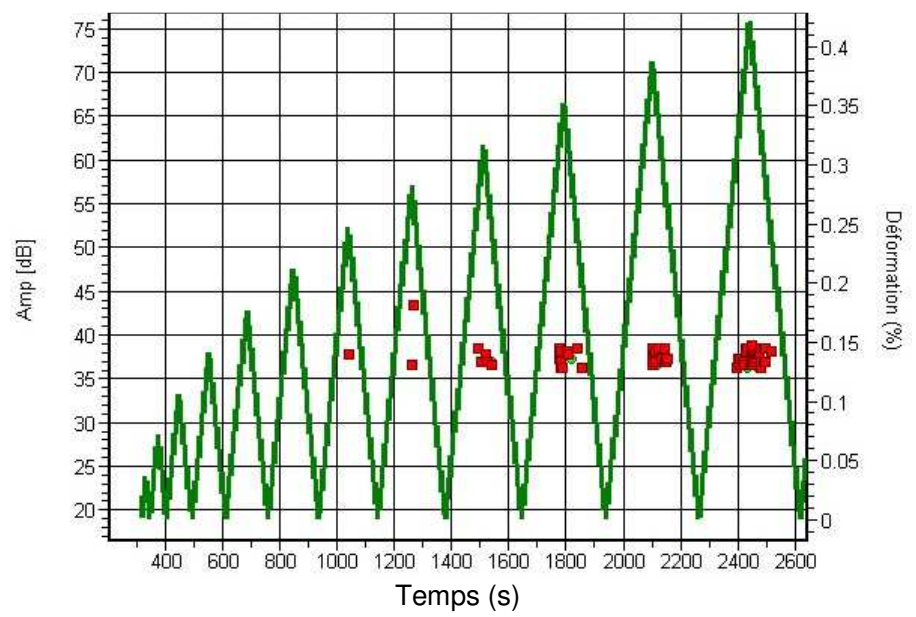

Figure 5 : Evolution du nombre d'alternances en chargement - déchargement

Le scénario décrit parait conforté par les résultats de la Figure 6, qui met en évidence la présence d'un mécanisme d'endommagement majeur que l'on attribue aux différents types de ruptures matricielles (ruptures longitudinales et transverses aux fibres), dont l'amplitude est comprise entre 35 et $45 \mathrm{~dB}$. Avec l'accroissement de contrainte, les fibres commencent à casser. Ceci peut être constaté sur la courbe de chargement par la sortie de la proportionnalité. Au-delà la limité d'élasticité, le nombre de fibres rompues devient important et l'occurrence de la charge maximale traduit la rupture quasi-totale des fibres sur la face en traction. Au-delà de la contrainte maximale, la fissuration procède en mode II, le long des fibres. La multiplicité des sources d'EA apparaît clairement sur la Figure 6, où l'on observe un spectre d'amplitude très large, allant jusqu'à $95 \mathrm{~dB}$.

En résumé, les séquences d'endommagement proposées sont au nombre de 4. Elles permettent de bien appréhender le comportement mécanique du matériau. Chacune est dominée par un mécanisme particulier (ceci n'exclut pas des chevauchements entre plusieurs mécanismes prenant place concomitamment) :

- La première phase commence au début de l'essai et se termine à la contrainte d'enregistrement des premiers événements d'émission acoustique. Cette partie est en principe exempte de tout endommagement.

- La seconde phase, localisée entre le début de l'endommagement $\left(\varepsilon_{\mathrm{EA}}=\right.$ $0,22 \%$ ) et la fin de proportionnalité. Cette phase met en évidence un endommagement matriciel multiple, avec quelques fibres rompues

- La $3^{\text {ème }}$ phase débute à la fin de proportionnalité et se termine à la contrainte maximale. Elle est caractérisée par des ruptures progressives des fibres en nombre important. La fin de cette partie est atteinte dès qu'un nombre critique de fibres se rompt à charge maximale 
- La dernière phase est constituée par une progression en mode II de la fissuration, par délaminage.

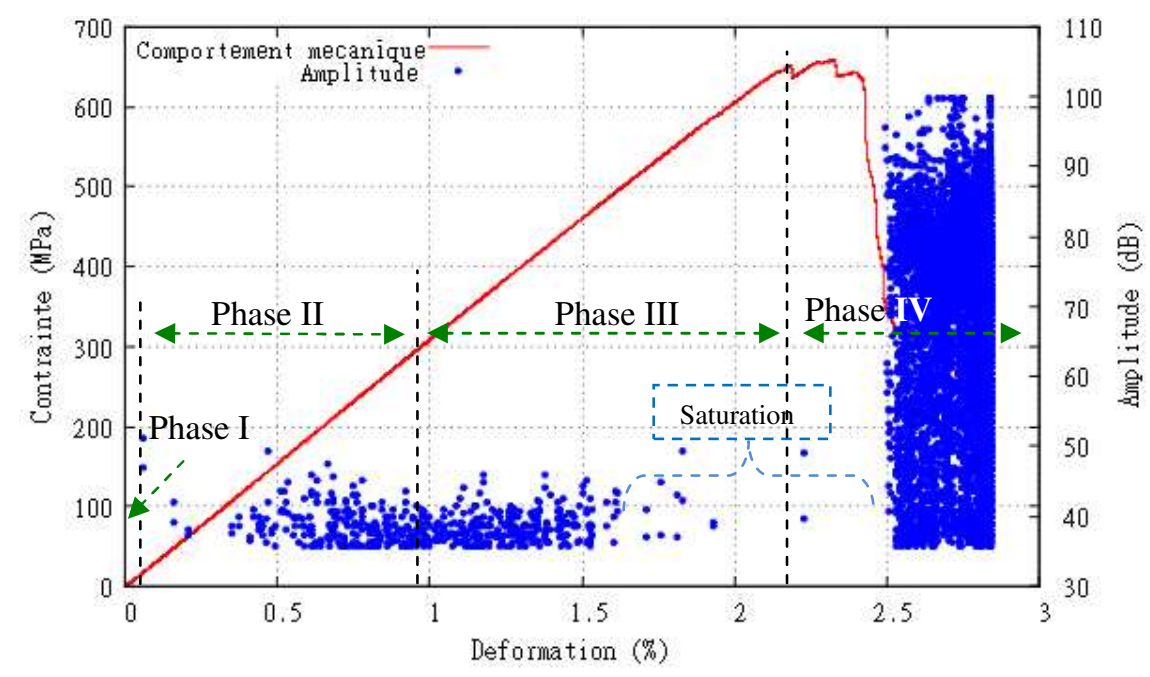

Figure 6 : Evolution de l'amplitude durant l'essai de flexion

Les enregistrements des événements d'EA a permis de localiser la concentration des émissions acoustiques, durant l'essai de flexion (Figure 7). Quelque soit le seuil utilisé, ce paramètre arrive à nous indiquer le point du début de la rupture et également le sens de la rupture. Le début d'endommagement est localisé au milieu de l'éprouvette. Au fur et à mesure que la charge augmente, la localisation des événements d'EA s'étend sur toute l'éprouvette. Le mode de rupture constaté sur les éprouvettes est de type II.

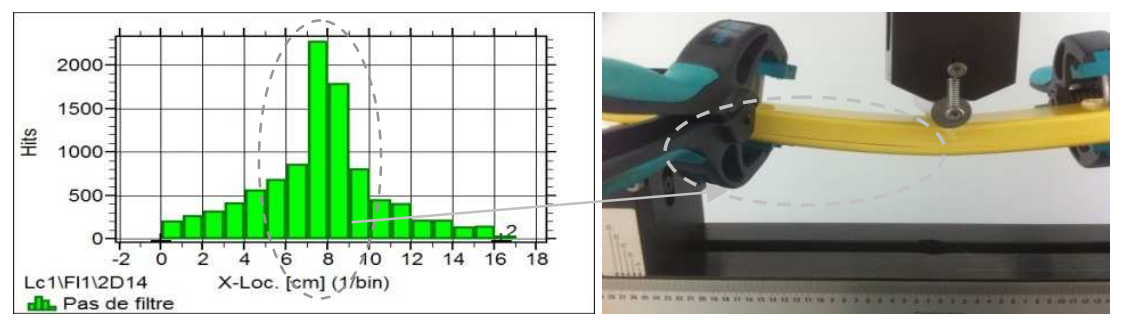

Figure 7 : Localisation de déformation dans un composite à matrice vinylester

Lors d'un cyclage de chargement - déchargement, à un niveau de contrainte donné (qu'il est utile de préciser), un matériau composite peut recommencer à s'endommager lors du rechargement à un niveau de contrainte inférieur à celui du déchargement précédent. Usuellement, tant que l'activité acoustique réapparait au- 
delà du point de déchargement précédent (suivi du rapport Felicity), le matériau est dit vérifier l'effet Kaiser.

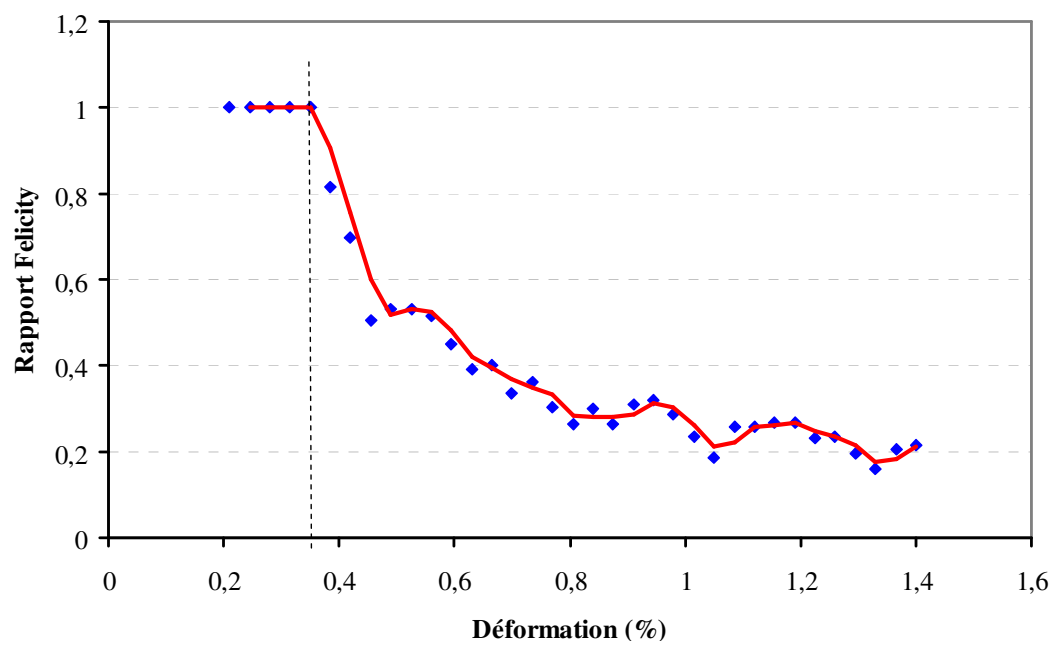

Figure 8 : Évolution du rapport Felicity en fonction de la déformation du matériau

La Figure 8 représente le rapport Felicity en fonction de la déformation du matériau. On remarque que l'effet Kaiser n'est plus vérifié à partir d'une déformation de $0,35 \%$. Ce résultat montre donc que le matériau continue à s'endommager même en phase de déchargement à valeur de déformation très faible et à l'intérieur du domaine de proportionnalité. La faible contrainte à rupture de la matrice et donc son endommagement important, qui intervient à faible niveau de contrainte (Figure 9a), son faible taux volumique sont des causes potentielles de la disparition de l'effet Kaiser à faible valeur de déformation. Par exemple, la matrice en s'endommageant fortement transversalement et longitudinalement doit créer des débris qui empêchent l'ouverture et la fermeture des microfissures lors des phases successives de chargement et de rechargement des cycles. Un facteur aggravant mis en exergue est l'hétérogénéité structurale de ce matériau composite obtenu par pultrusion, où l'on observe souvent des zones de prédominance de fibres et/ou de matrice (Figure 9b). La rupture massive des fibres engendre l'activation concomitante de multiples mécanismes d'endommagement. Le réarrangement de ces mécanismes lors du rechargement s'accompagne d'une activité acoustique importante à des niveaux de chargement inférieurs à ceux du déchargement précédents). 


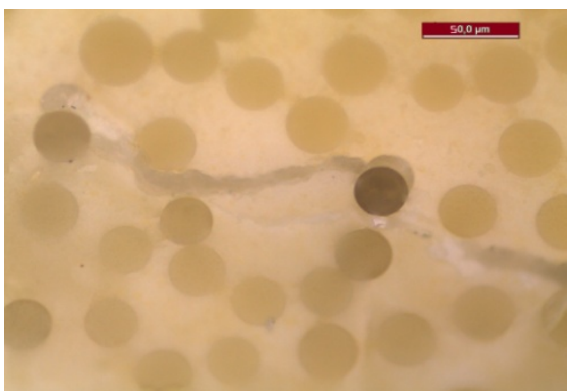

(a)

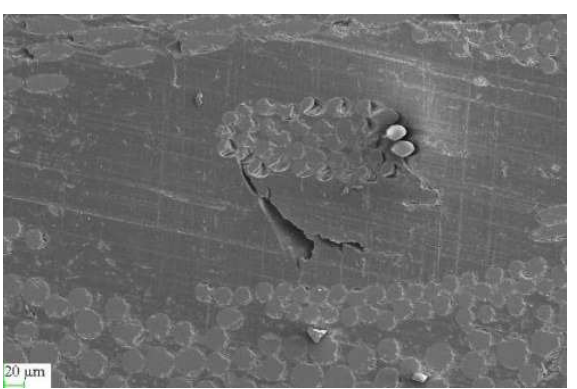

(b)

Figure 9 : (a) Illustration de la fissuration matricielle au sein du matériau composite étudié ; (b) Il faut également noter les hétérogénéités structurales : zones riches en fibres et celles riches en matrice présentes dans le matériau.

\subsection{Fatigue en flexions trois points}

La Figure 10 présente l'évolution de l'activité acoustique durant un essai de fatigue par paliers. On constate une absence d'EA durant le premier palier de fatigue. Puis, une forte activité s'installe dés le début du deuxième palier et continue jusqu'à la fin du palier 3 , avec une variation d'amplitude entre $35 \mathrm{~dB}$ et $50 \mathrm{~dB}$. Ceci est cohérent avec les essais quasi-statique, dans la mesure où le mécanisme dominant est relatif aux ruptures de la matrice que ce soit dans le sens longitudinal (parallèle aux fibres) ou transverse.

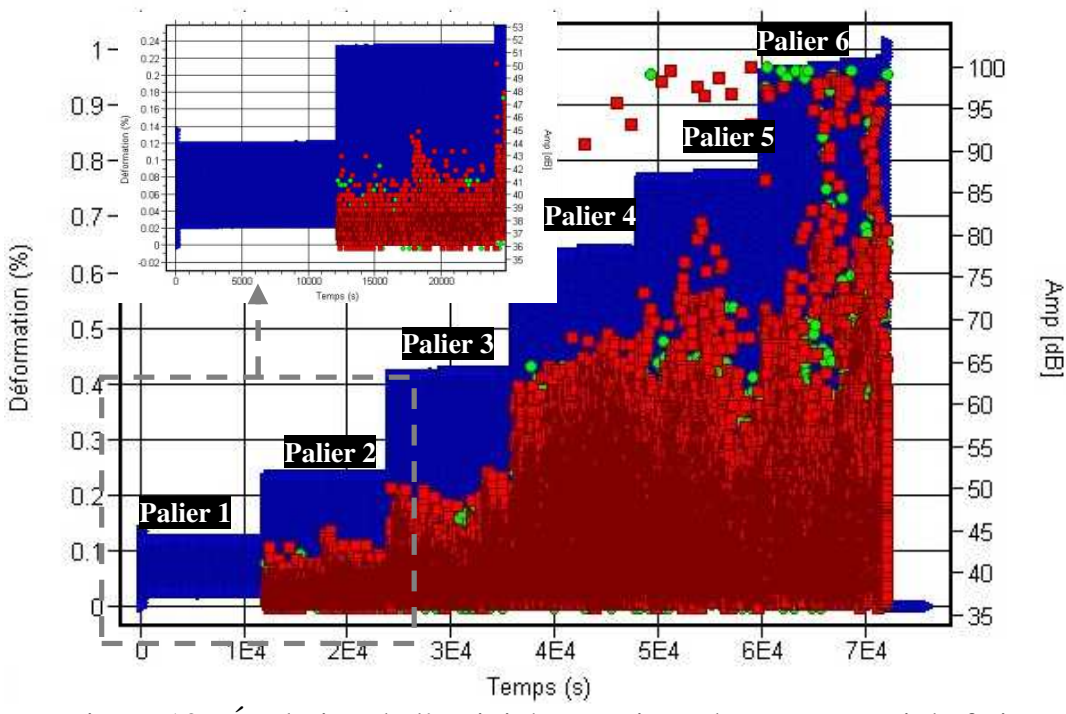

Figure 10 : Évolution de l'activité acoustique durant un essai de fatigue 
Dans le palier 4, on remarque l'enregistrement d'amplitudes élevées (allant jusqu'à 90dB). Ceci peut s'expliquer en considérant qu'avec la cyclicité de la contrainte, les frottements entre fibres et débris de matrice induisent des ruptures de fibres en quantité appréciable. Il peut s'agir, en particulier, des fibres les plus altérées, ou bien celles possédant les plus faibles diamètres. Cette explication est confortée par des amplitudes d'événements d'EA, allant jusqu'à $75 \mathrm{~dB}$. Au-delà de ce palier, l'on remarque un spectre large d'amplitude d'événements acoustiques; ce qui correspond à la coexistence de tous les mécanismes d'endommagent usuellement présents dans un matériau composite unidirectionnel. En particulier, la rupture en masse des fibres de verre sont reflétées par des amplitudes élevées d'événements d'EA.

Si on s'intéresse à la localisation des événements acoustiques (Figure 11), on relève une concentration d'EA dans la moitié d'éprouvette, proche du capteur 2.

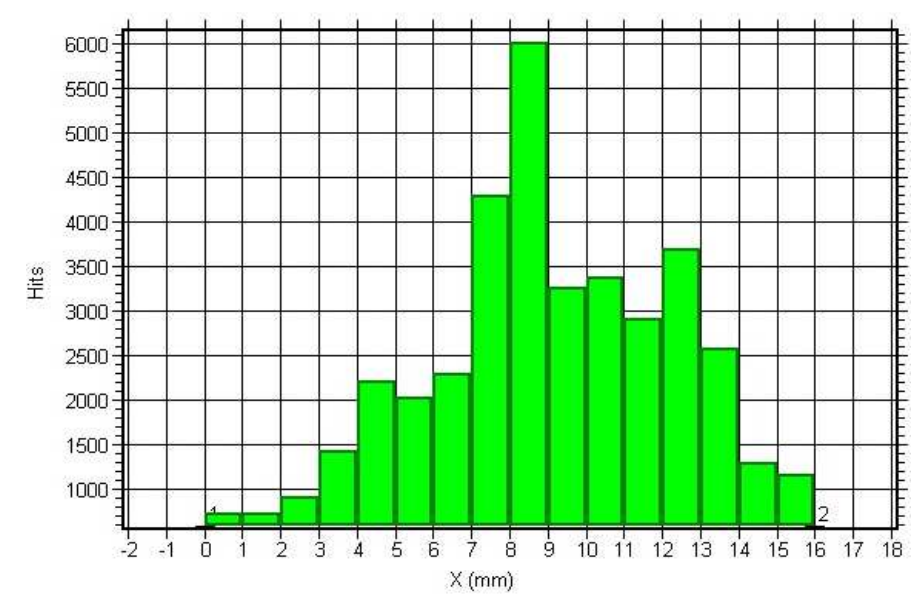

Figure 11 : Evénements acoustiques localisés, durant un essai de fatigue

\section{Conclusions}

Le couplage entre les différents types d'essai en flexion trois points (monotone, cyclique, fatigue) et les enregistrements d'émissions acoustiques ont permis de déceler la contrainte du premier endommagement des composites. Ce qui a permis de définir l'intervalle $\left[\sigma_{\text {Début d'endommagement }}, \sigma_{\text {Fin de linérité }}\right]$ dans lequel les essais de fatigue seront pratiqués ultérieurement, afin de caractériser finement le comportement des matériaux étudiés.

A l'aide d'une analyse conjuguée des résultats d'essais mécaniques, des enregistrements et des traitements des activités d'émission acoustique, il a été possible de construire un scénario cohérent, décrivant l'endommagement et la ruine 
de ce matériau composite unidirectionnel. Bien évidemment, la technique d'EA, malgré sa grande sensibilité, laisse toujours des zones d'ombre. Ce qui incite à la prudence, lors de l'analyse et la comparaison des résultats bibliographiques : Les fibres ne sont identiques, les matrices ne le sont pas également, les conditions de mise en œuvre, le processus d'endommagement n'est pas systématiquement récurrent, la concomitance des mécanismes d'endommagement à niveau de contrainte élevée, les émissions acoustiques parasites sont autant de raisons qui font que la technique d'EA, appliquée aux matériaux composites, doit certainement apporter une argumentation qualitative. L'exploitation quantitative des résultats doit être, quant elle, réalisée avec une très grande prudence.

\section{Remerciements}

Ce travail a été conduit au sein du projet DECID2, financé par les fonds FUI (Fonds Uniques Inter - ministériels), de la DGE. MDH souhaite remercier ces fonds, ainsi que la Région Pays-de-La-Loire pour le soutien financier. Les auteurs souhaitent également remercier, Mme Annick Perronet, pour les observations en microscopie électronique à balayage.

\section{Bibliographie}

Binse P., «Fabrication de profilés en composites par pultrusion », Technique de l'ingénieur, A3730.

Carmona V., "Étude de l'endommagement de matériaux composites par tomographie $X$ et émission acoustique », rapport de thèse, INSA de Lyon, 2009.

Crouzeix V., «Identification de champs de propriétés mécaniques de structures composites à partir de mesures de champs de déplacements », rapport de thèse, Université Toulouse III - Paul Sabatier, 2008.

Drissi-Habti M., "Damage development and moduli reduction in a unidirectional SiCMAS.L composite », Scripta Metallurgica \& Materialia, Vol. 33, nº, pp. 967-973, 1995

Ducret D., "Elasticité anisotrope et endommagement des matériaux composites : caractérisation ultrasonore et modélisation micromécanique », rapport de thèse, INSA de Lyon, 2000.

Huguet S., «Application de classificateurs aux données d'émission acoustique : identification de la signature acoustique des mécanismes d'endommagement dans les composites à matrice polymère », Rapport de thèse, INSA de Lyon, 2002.

Kim S. T., Lee Y. T., « Characteristics of damage and fracture process of carbon fiber reinforced plastic under loading -unloading test by using AE method »,Materials science and engineering, Vol. A234-236, pp. 322-326, 1997.

Kotsikos G., Evans J. T., Gibson A. G., Hale J., «Use of acoustic emission to characterize corrosion fatigue damage accumulation in glass fiber reinforced polyester laminates », Polymer composites, Vol. 20, pp. 689-696, 1999. 